\title{
Low-energy electron collisions with the alanine molecule
}

\author{
Milton M. Fujimoto ${ }^{1, a}$, Jonathan Tennyson ${ }^{2}$, and Sergio E. Michelin ${ }^{3}$ \\ 1 Departamento de Física, Universidade Federal do Paraná, 81531-990 Curitiba, PR, Brazil \\ 2 Department of Physics and Astronomy, University College London, Gower St., London, WC1E 6BT, UK \\ 3 Departamento de Física, Universidade Federal de Santa Catarina, 88040-900 Florianópolis, SC, Brazil
}

Received 29 October 2013 / Received in final form 29 January 2014

Published online 25 March 2014

(c) The Author(s) 2014. This article is published with open access at Springerlink.com

\begin{abstract}
A theoretical study on elastic electron collisions with two conformers of amino acid alanine $\left(\mathrm{CH}_{3} \mathrm{CH}\left(\mathrm{NH}_{2}\right) \mathrm{COOH}\right)$ is reported. Differential and integral cross sections are computed for collision energies in the 1-10 eV range. The UK molecular R-matrix codes are used to compute scattering amplitudes within the static exchange plus polarization (SEP) approximation. Both alanine conformers have large permanent dipole moment so to calculate cross sections the Born closure procedure is included to take into account long-range interactions. Comparisons of calculated differential cross sections with available data for glycine are made and display certain similarities. Two shape resonances are detected for each conformer: a narrow one located at $2.7 \mathrm{eV}$ and $3.5 \mathrm{eV}$ which is probably associated with the unoccupied $\pi^{*}$ orbital of the carboxyl group, and a broader resonance at $8.6 \mathrm{eV}$ and $9.8 \mathrm{eV}$.
\end{abstract}

\section{Introduction}

When ionizing radiation interacts with living tissue, high energy electrons are generated, and these electrons interact with their surroundings generating secondary electrons with lower energies in a cascade effect. In a landmark paper, Boudaïffa et al. [1] demonstrated the importance of these secondary, low-energy electrons, with energy less than $20 \mathrm{eV}$, in causing severe damage to DNA molecules. It has been suggested that most of the damage in DNA, up to $70 \%$, is caused by these secondary, low-energy electrons or ions [2]. The mechanism for this DNA damage is dissociative electron attachment (DEA): basically a low-energy electron is captured by a molecule producing a temporary anion, a resonant state, which can decay into neutral and/or negative ion fragments. It has been shown that this process can induce single or double strand breaks in DNA molecules, depending on which DNA constituent absorbs the low-energy electron and what energy the electron has. These observations have stimulated significant interest in electron-molecule collisions with small biomolecules and the constituents of larger one, with particular emphasis on characterizing the resonance states of these systems. There are many studies of electron collisions with DNA building blocks [3-9] such as amino acids [10-15], nucleobase [16-33], sugar and phosphate moieties [34-43].

\footnotetext{
* Contribution to the Topical Issue "Electron and Positron Induced Processes", edited by Michael Brunger, Radu Campeanu, Masamitsu Hoshino, Oddur Ingólfsson, Paulo Limão-Vieira, Nigel Mason, Yasuyuki Nagashima and Hajime Tanuma.

a e-mail: milton@fisica.ufpr.br
}

$L$ - $\alpha$-alanine is one of the simplest $\alpha$-amino acids and is one of the building blocks of natural proteins. Indeed it is one of 20 amino acids used to encode the genetic code. This molecule can therefore be considered a model system for more complex molecules, such as DNA constituents, because it has a carbon backbone as well as carboxyl and amino groups. Alanine also is important for radiation dosimetry as it is used as a secondary standard in high dose dosimetry [44-46]. In the solid state, $L$ - $\alpha$-alanine generates stable radicals which are suitable for use in electron paramagnetic resonance (EPR) dosimetry [47].

There have been only limited studies on electron collisions with the alanine molecule. The first study of gasphase alanine DEA used mass spectroscopy with a high resolution electron energy monochromator [48]. Negative ions were analysed and it was observed that alanine has several features in common with the amino acid glycine, specifically regarding the possibility of attachment of an electron to the unoccupied $\pi^{*}$ orbital of the carboxyl group $(-\mathrm{COOH})$. This work was extended by Ipolyi et al. [49], who studied the appearance energies of the positively charged fragments induced by the electron impact ionization and dissociative electron impact ionization of gas phase alanine. Using mass spectroscopy they observed that the value of the ionization energy for the parent ion with $m / Z=89$ was $9.12 \mathrm{eV}$. The most important positive fragments obtained were ions with $m / Z$ of 74,44 and 28 and appearance energies of $10.74,9.10$ and $10.85 \mathrm{eV}$, respectively. Total dissociative electron attachment cross sections have been measured for the amino acids glycine, alanine, proline, phenylalanine, and tryptophan, at energies below the first ionization energy by Scheer et al. [50]. 
These authors observed an increase in the cross section at $1.2 \mathrm{eV}$ which, following suggestions from other authors, they attributed to attachment via the $\pi^{*}$ orbital of - $\mathrm{COOH}$ group. They also suggested that direct attachment to the $\sigma^{*}$ orbital may be possible.

The first measured elastic differential cross section (DCS) for alanine was presented by Marinković et al. [51] for energies ranging between $20 \mathrm{eV}$ and $80 \mathrm{eV}$. A set of cross section were computed using a corrected independent-atom method incorporating an improved quasi-free absorption model and the measured data set was normalized to these calculations. Integral elastic, inelastic and total cross sections were also computed for impact energies ranging from 1 up to $10000 \mathrm{eV}$. Abouaf [52] studied excited electronic states of glycine and $D$-alanine in the gas phase using electron energy loss spectroscopy (EELS) and obtained good agreement with recent theoretical calculations [53]. Abouaf [52] also presented DEA cross sections with a series of dips at some energies which were interpreted as due to competition between resonance decay channels.

Electron attachment in clusters of glycine, alanine and serine amino acids inside helium nanodroplets was observed by da Silva et al. [54]. For glycine and alanine, the results recorded in these clusters showed that the major product is parent anions which contrasts with the results for the monomer, where dehydrogenated anions $(\mathrm{A}-\mathrm{H})^{-}$ are the major product. Panosetti et al. [10] calculated total elastic cross sections and resonance features for gas phase glycine, alanine, proline and valine at their equilibrium geometries. They studied the one-dimensional dissociation path of transient negative ions following resonant state formation and concluded that it is not possible to explain the detachment of - $\mathrm{OH}$ using a simple one-dimensional model in these complex molecules.

In this paper we report theoretical rotationallyunresolved elastic electron scattering cross sections for two different conformers of alanine in the gas phase at impact energies ranging from $1 \mathrm{eV}$ to $10 \mathrm{eV}$. Given the importance of alanine, as represented by the issues discussed above, it is surprisingly that there appears to be no published calculated or measured elastic differential cross section for this molecule at energies below $10 \mathrm{eV}$. We have therefore calculated elastic DCS including both polarizations effects and long-range interactions due to large permanent dipole moment of alanine. The influence of polarization effects on the resonance feature is analysed.

The organization of article is as follows: Section 2 presents an outline of the theory and some details of the calculations are provided in Section 3. Section 4 presents and discusses our calculated data; this is followed by a summary of conclusions.

\section{Calculations}

\subsection{The R-matrix method}

The UK molecular R-matrix methodology used in this work is well-established and described in detail elsewhere $[55,56]$. Here we just present the most important features of the method necessary to understand the discussion.

In the R-matrix method, the configuration space is divided in two regions: inner and outer. The inner region is limited by a sphere of radius $a$ which should contain all electronic density of the $N$-electrons of the isolated target. In this region, the $N+1$ electrons have short-range interactions and a precise description is desirable as the physics here is richer than the outer region because of the correlation and exchange interactions. In the inner region it is necessary calculate the electronic structure of the $(N+1)$-electron complex only once per symmetry, which is advantageous since most of the computational effort and time are expended here.

In the outer region, the scattering electron interacts with the $N$-electrons of the target via long range potential interactions, such as polarization or dipole interactions, and no correlation and exchange effects are included. In this region, the scattering electron can be represented by a one-electron wave function which moves in a scattering potential described by the electronic density of the target.

In the inner region, the $(N+1)$-electron wave function is described by:

$$
\begin{aligned}
\Psi_{k}^{N+1}\left(x_{1} \ldots x_{N+1}\right)= & \mathcal{A} \sum_{i j} a_{i j k} \phi_{i}^{N}\left(x_{1} \ldots x_{N}\right) u_{i j}\left(x_{N+1}\right) \\
& +\sum_{i} b_{i k} \chi_{i}^{N+1}\left(x_{1} \ldots x_{N+1}\right)
\end{aligned}
$$

where $\phi_{i}^{N}$ is the target wave functions of the $i$ th state and $u_{i j}$ is an orbital used to represent the continuum electron with a partial wave expansion up to some maximum value of $\ell, \ell_{\max } ; \mathcal{A}$ is an antisymmetrization operator introduced so that the indistinguishable inner-region electrons satisfy the Pauli principle. The second summation in equation (1) contains functions $\chi_{i}^{N+1}$ which describe all $N+1$ electrons but vanish at $r=a$; thus these are described as $L^{2}$ configurations. They are included to relax the constraint of orthogonalization between scattering and target orbitals of the same symmetry, and, as discussed below, to allow for target polarization effects. $a_{i j k}$ and $b_{i k}$ are the variationally-optimized coefficients of expansions.

In our inner region calculations, polarization effects are included via second sum in equation (1) using singlyexcited $L^{2}$ configurations of the Hartree-Fock (HF) ground state wave function. This is achieved by promoting one target electron into a virtual (unoccupied target) orbital and simultaneously also placing the scattering electron into a virtual orbital generating a two-particle one-hole $(2 p, 1 h)$ configuration. This model is usually denoted static exchange plus polarization (SEP).

In the outer region, a one-particle coupled secondorder differential equation for the continuum electron must be solved for the electron scattering process. The $\mathrm{R}$-matrix is used to match inner and outer solutions. The $\mathrm{R}$-matrix constructed on the boundary is propagated to large $r$ and then used to obtain K-matrices which are used to compute the scattering observables. Alanine has 


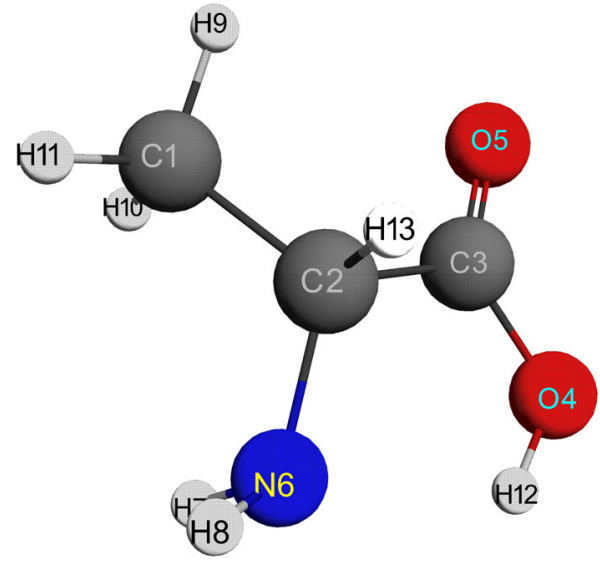

Fig. 1. Chemical structure of the $L$ - $\alpha$-alanine conformer $\mathrm{M}$ of amino acid alanine. Gray, red and blue spheres are carbon, oxygen, and nitrogen atoms, respectively, while the smaller white spheres are hydrogen (figure generated by MacMolplt [65]).

a large permanent dipole, thus a Born closure procedure is used to compute the effect of the long-range interaction neglected when only partial waves from 0 to $\ell_{\max }$ are considered. In this procedure our low- $\ell \mathrm{T}$-matrices are added to analytic dipole Born T-matrices, using the ANR approximation to take account of rotational motion, and then corrected by subtracting the partial wave dipole Born contributions [57-59]. The rotationally-unresolved elastic differential cross sections are calculated using the code POLYDCS [60], which also computes rotationally-resolved elastic and inelastic cross section which are then summed until convergence is achieved. Since it is not possible to study rotationally resolved cross sections experimentally for molecules of the size of alanine, only rotationally unresolved cross sections are presented here.

In this work, initial calculations were performed using version 4.1 of the Quantemol-N expert system [61], with more detailed studies being performed directly with the UKRMol codes [62]. Quantemol-N provides a quick way constructed correct and self-consistent inputs the UKRMol code but the options available do not encompass all possible scattering models that one may wish to test. It is therefore our practice to start from Quantemol-N calculations and adjust these inputs to performing detailed tests if this is thought necessary.

\subsection{Calculation details}

One of the conformers studied is the lowest energy conformer, called conformer I, and the geometry used here is the same described by Blanco et al. [63]. The second conformer of $L$ - $\alpha$-alanine studied here is based on the nuclear geometry described on the NIST website [64]. This conformer is closer to the conformer IIb described by Blanco et al. [63]. For simplicity, this conformer is called conformer M below; its structure is shown in Figure 1.

Alanine is a closed shell system with no symmetry so its ground electronic state is ${ }^{1} A$. Both conformers were described at the Hartree-Fock level using 6-31G* basis set and the permanent dipole moment obtained for conformer $\mathrm{I}$ is $1.51 \mathrm{D}$ and $5.49 \mathrm{D}$ for conformer M. These are very close to the expected dipole moment values of 1.4 D for conformer I and 5.45 D for conformer IIb [63]. The dipole moment of conformer $\mathrm{M}$ is similar to the calculated dipole of conformer II and the experimental value measured for conformer III by Godfrey et al. [66].

To calculate scattering properties we have used both 6-31G* and ccp-VTZ basis set to represent the target. However, the results with these two basis sets are essentially the same and, as ccp-VTZ is computationally more expensive, we present results for the $6-31 G^{*}$ basis set. For the $L$ - $\alpha$-alanine conformer $\mathrm{I}$, the rotational constants used in POLYDCS to calculate rotational excitation cross sections were $A=0.0210261 \mathrm{meV}, B=0.0128418 \mathrm{meV}$ and $C=0.0094067 \mathrm{meV}$ and for the conformer $\mathrm{M}$ the rotational excitation cross sections were $A=0.0205562 \mathrm{meV}$, $B=0.0143724 \mathrm{meV}$ and $C=0.0089903 \mathrm{meV}$. Our DCS results without Born correction can be considered rotationally unresolved as we sum over rotational transitions until the convergence is achieved. To represent long-range interactions due to large permanent dipole moment we have used Born closure procedure.

To define the boundary of the inner region, we tested the effect of varying the $\mathrm{R}$-matrix radius from $10 a_{0}$ to $15 a_{0}$. As we need a balanced basis sets to represent the continuum and the target inside the sphere, we chose $a=11 a_{0}$, although all values gave similar results. The continuum wave functions are expanded in GTOs using a partial wave expansion [67]. We tested the partial waves expansion by calculating cross sections including partial waves from 0 to $\ell_{\max }=4$ and $\ell_{\max }=5$. The results are essentially the same; however the $\ell_{\max }=5$ calculations showed non-physical behavior in DCSs for angles higher than $150^{\circ}$ after the Born closure was introduced. Therefore the results exhibited here are for $\ell_{\max }=4$ and completed with Born closure, unless otherwise specified.

To allow for polarization effects in the inner region, we employ up to 30 virtual orbitals from the target self consistent field (SCF) calculation to generate $(2 p, 1 h) L^{2}$ configurations; both singlet and triplet excited target states were considered. The number of virtual orbitals is more than sufficient to converge a static exchange (SE) calculation. In the outer region the long-range polarization potential could be included a term of the form $-\alpha / R^{4}[68]$. However we chose not to do this here since the permanent dipole moment of alanine is relatively large and dominates the long-range interactions even in the case of conformer I which dipole moment is $1.51 \mathrm{D}$.

\section{Results and discussion}

Figure 2 shows how our calculated eigenphase sums vary with the level of treatment of polarization for conformers I and $\mathrm{M}$ of alanine. The structure in the eigenphase sums indicates the possible presence of one or, with more polarization, two resonances. A SE calculation, which neglects polarization effects completely, places the lower resonance 

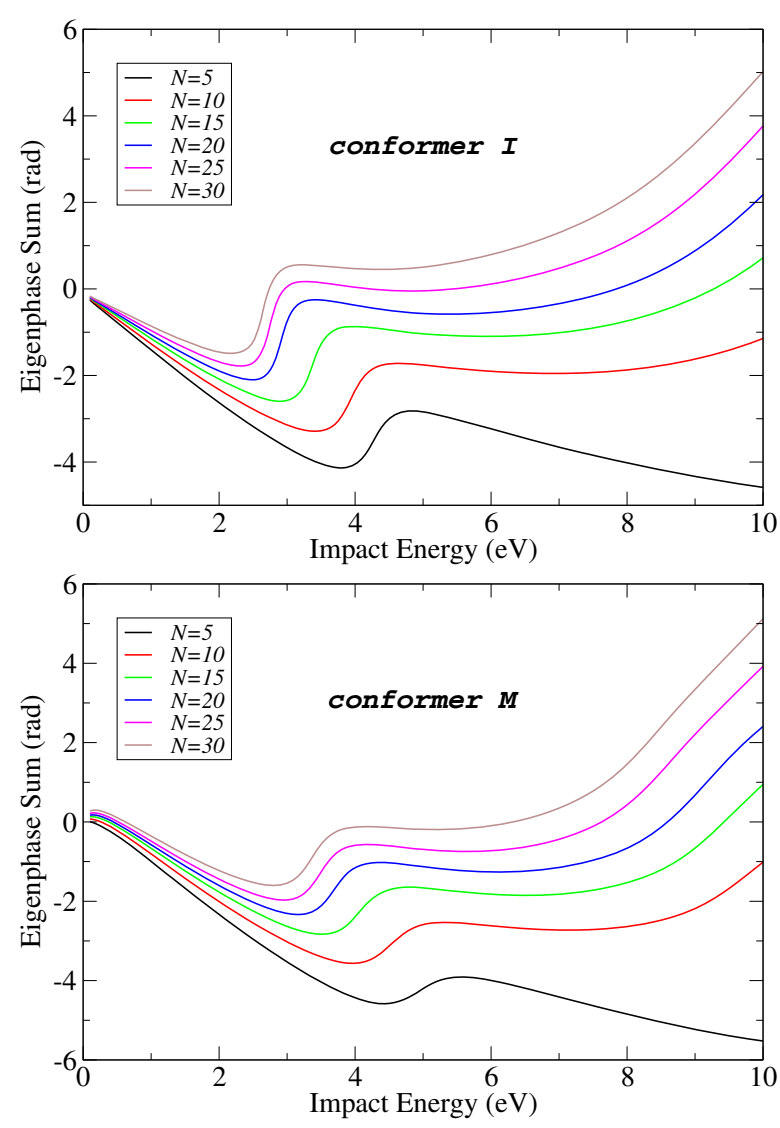

Fig. 2. Dependence of the eigenphase sums on the number of virtual orbitals $(N)$ used in SEP model calculations for electron scattering by conformers I and $\mathrm{M}$ of alanine.

feature at around $5 \mathrm{eV}$. As more polarization is included, this resonant state moves to lower energies. An automated fit to the eigenphase sums using the Breit-Wigner formula [69] gives the final resonance positions(width) for the conformer I at the $2.7(0.3) \mathrm{eV}$ and $9.8(2.5) \mathrm{eV}$. The resonance positions(width) for the conformer $\mathrm{M}$ are $3.4(0.6) \mathrm{eV}$ and 8.6(1.8) eV.

Figure 3 shows convergence of the elastic DCS with respect to polarization effects without Born closure for the conformer I, for $2 \mathrm{eV}$ and $10 \mathrm{eV}$. Conformer I has a smaller dipole moment than conformer M, so polarization effects are expected to exert more influence on the DCSs for conformer I than M. The results suggest that the DCS are reasonably well-converged when 30 virtual orbitals are retained to compute polarization, even if the eigenphase sums themselves may not be fully converged, especially going to higher energies. Our previous study on ethanol [70] showed that use of 35 virtual orbitals gives very well converged cross sections.

Figure 4 presents the elastic SEP-DCS including Born correction for I, which take into account the coupling partial wave with $\ell>4$ by the long-range potential. Inclusion of the high- $\ell$ contributions raises the DCS significantly and, as expected, causes them to be very strongly forward peaked. Under these circumstances the DCS is much less sensitive to the treatment of polarization than calculations
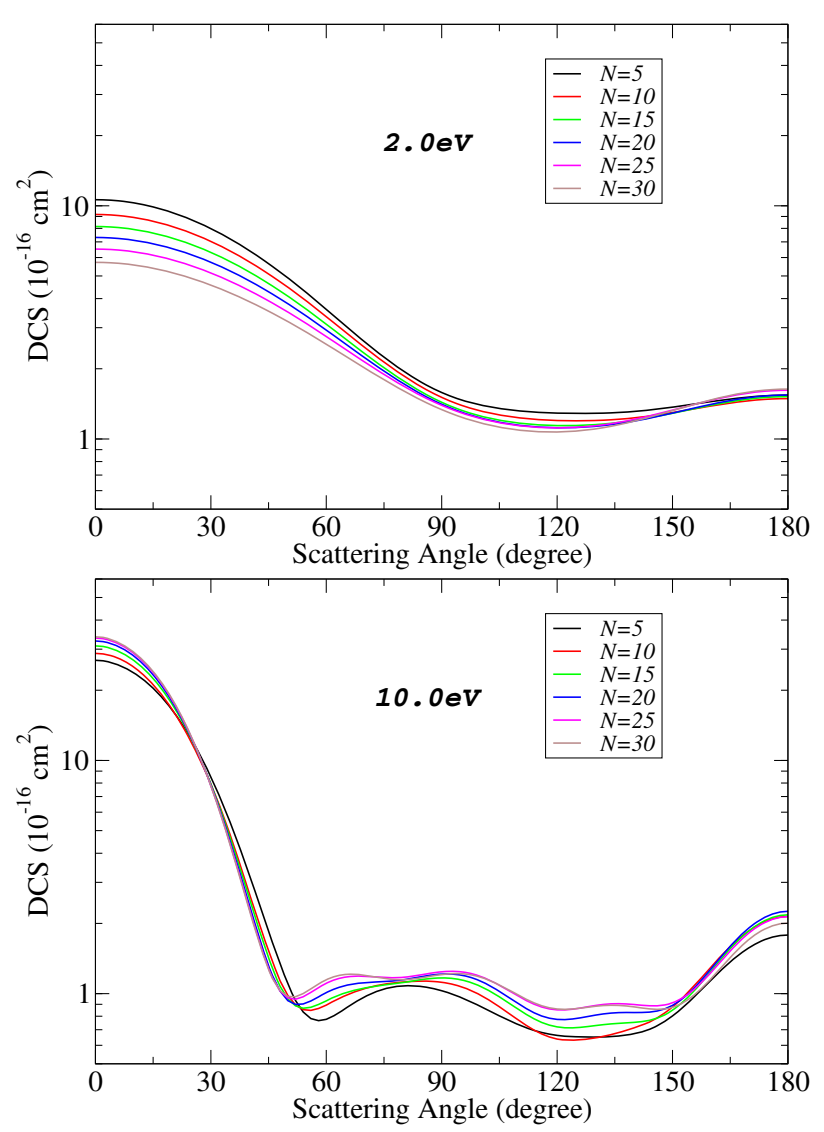

Fig. 3. Convergence of DCS with respect to number of virtual orbitals $(N)$ used in the calculation for impact energies of $2 \mathrm{eV}$ and $10 \mathrm{eV}$. Results for conformer I and do not include a Born correction.

which neglect the Born correction due, simply, to the magnitude of alanine's permanent dipole moment. This means that in practice the Born-corrected DCSs do not change significantly once 10 virtual orbitals are included in the calculation for the conformer M, figure not shown.

A summary of our final DCSs for both conformers in the energy range from 1 to $10 \mathrm{eV}$ is given in Figure 5 . Below $8 \mathrm{eV}$, the shape of the DCSs suggests a strong contribution of the $d$-partial waves which can be associated with the minimum around $130^{\circ}$. For conformer M, the DCS increases at lower energies meaning that for $1 \mathrm{eV}$ the DCS is larger by a factor of at least 5 than the DCS at $10 \mathrm{eV}$. This again is a consequence of alanine's large dipole moment. For conformer I, the difference between 1 to $10 \mathrm{eV}$ is not so marked because its dipole is relatively small.

There are no previous theoretical or experimental DCSs at energies lower than $10 \mathrm{eV}$ available for us to compare with. However we can compare our results with those available results for glycine. Alanine and glycine have very similar structures, both have carboxylic group $(-\mathrm{COOH})$, amino group $\left(-\mathrm{NH}_{2}\right)$. Alanine is slightly larger as it corresponds the substitution of one $\mathrm{H}$ atom from the $-\mathrm{CH}_{2}$ group in glycine by a $-\mathrm{CH}_{3}$ group. It is possible to get various different conformers with the same sequential 

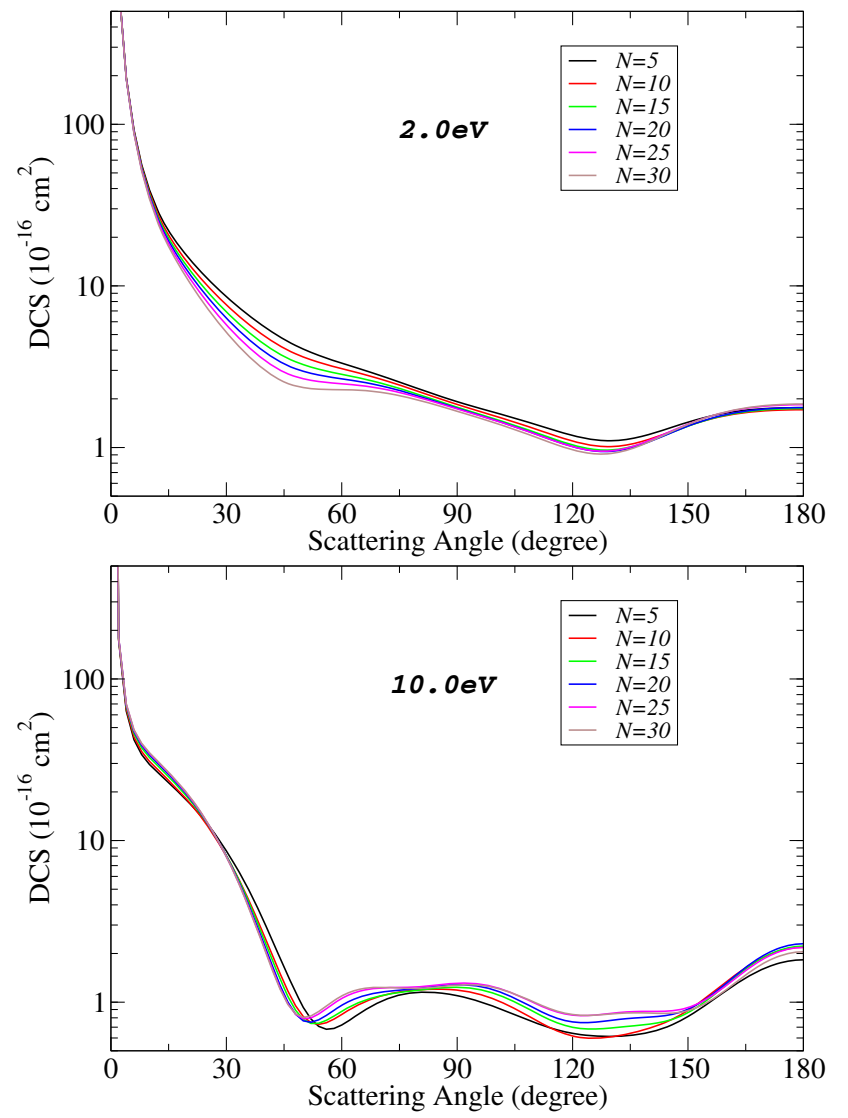

Fig. 4. Convergence of Born-corrected DCS with respect to number of virtual orbitals $(N)$ used in the calculation for impact energies of $2 \mathrm{eV}$ and $10 \mathrm{eV}$. Results for conformer I.

arrangement of atoms by only rotating or changing angles of chemical bonds. Figure 6 compares our DCS for conformers I and M (with no Born correction) with available data for the lowest energy conformer of glycine from Tashiro [71] and dos Santos et al. [72]. The results for glycine do not include a Born correction. At the low impact energies it is possible to see clearly differences in the DCS between alanine conformers I and M caused partially by the different arrangement of atoms, but mainly due to the difference in magnitude between the dipole moments. As the impact energy increases, the DCSs become similar. While the permanent dipole moment of alanine conformer I $(1.51 \mathrm{D})$ is similar to the glycine which is $1.32 \mathrm{D}$ [72], conformer $\mathrm{M}$ has a much bigger dipole moment $(5.49 \mathrm{D})$. Despite the differences in molecular size and dipole moments, these three molecules have very similar DCSs. Even without the Born correction, the DCSs for conformer $\mathrm{M}$ has a more pronounced peak at forward angles than glycine at all impact energies considered. However above $50^{\circ}$, the alanine conformer $\mathrm{M}$ results are generally close in magnitude to those available for glycine.

Figure 7 shows how our calculated integral cross sections (ICS) depend on polarization effect (number of virtual orbitals) for both conformers, without Born correction just to emphasize the resonance features. It is well-known that molecules containing - $\mathrm{COOH}$ group
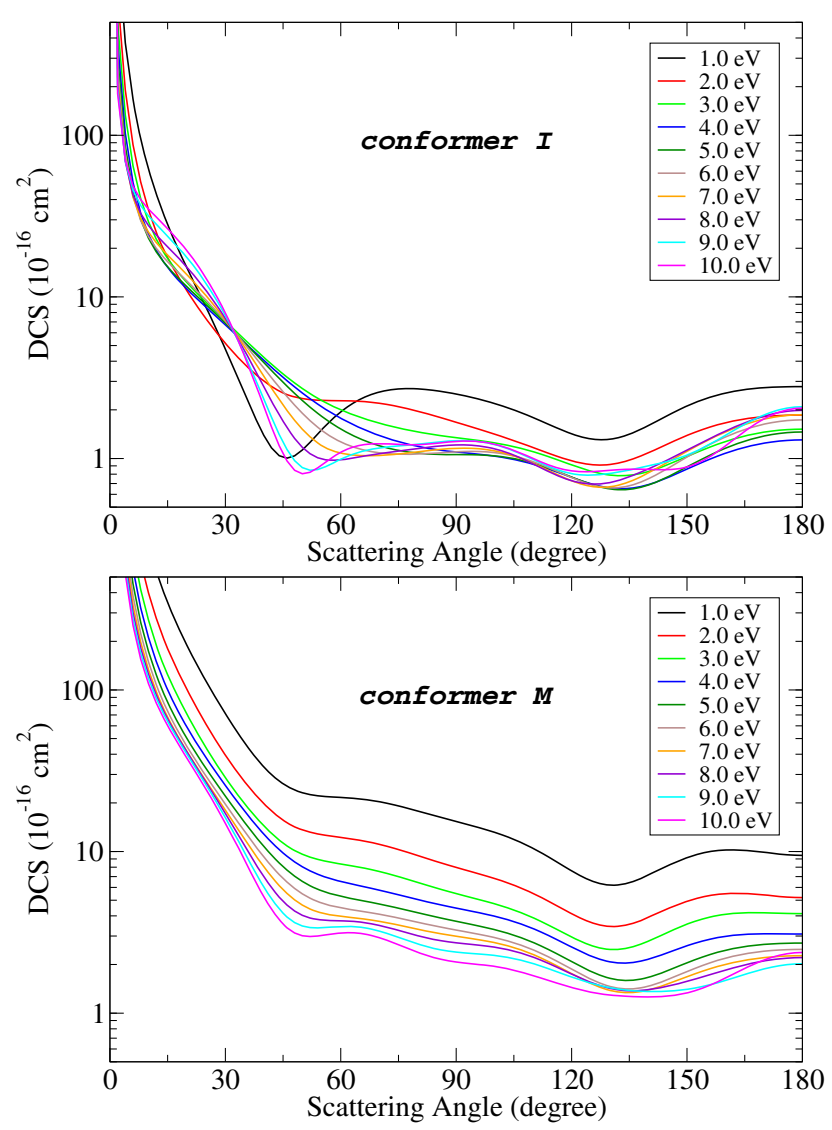

Fig. 5. DCS for elastic electron scattering by alanine conformers I and M using an SEP model with 30 virtual orbitals and a Born correction. The DCS decreases with impact energies, given in steps of $1 \mathrm{eV}$, from $1 \mathrm{eV}$ (highest) to $10 \mathrm{eV}$ (lowest).

often show $\pi^{*}$ resonance features in low-energies electron collisions [73]. Our calculations give a sharp, low-energy resonance peak at $2.7 \mathrm{eV}$ and $3.4 \mathrm{eV}$ for conformers I and $\mathrm{M}$ respectively, which are in good agreement with the resonance energy around $3 \mathrm{eV}$ calculated for various amino acids, such as, glycine, alanine, proline and valine, by Panosetti et al. [10]. For comparison, our alanine conformer $\mathrm{M}$ resonance position is the same as that obtained by Tashiro [71] for glycine using a similar model of polarization to the one we use here. The resonance position for conformer I is in good agreement of $2.3-2.8 \mathrm{eV}$ obtained for glycine by dos Santos et al. [72].

There are various studies indicating that the $\pi^{*}$ resonance can be related to the DEA peak products at electron impact energy near $2 \mathrm{eV}$ for amino acids. Aflatooni et al. [73] used electron transmission spectroscopy to measure vertical attachment energies for the formation low-lying temporary anion states of alanine at $1.80 \mathrm{eV}$ and suggested that the origin of these anions is the temporary occupation of $\pi^{*}$ vacant orbital of carboxyl group. Ptasińska et al. [34] studied DEA in the alanine using high resolution spectroscopy and observed low-energy DEA peaks at $1.27,1.42,1.7,2.7$ and $3.2 \mathrm{eV}$; they associated all these peaks with the $\pi^{*}$ orbital of carboxyl group. They observed further resonance peaks at higher energies, 

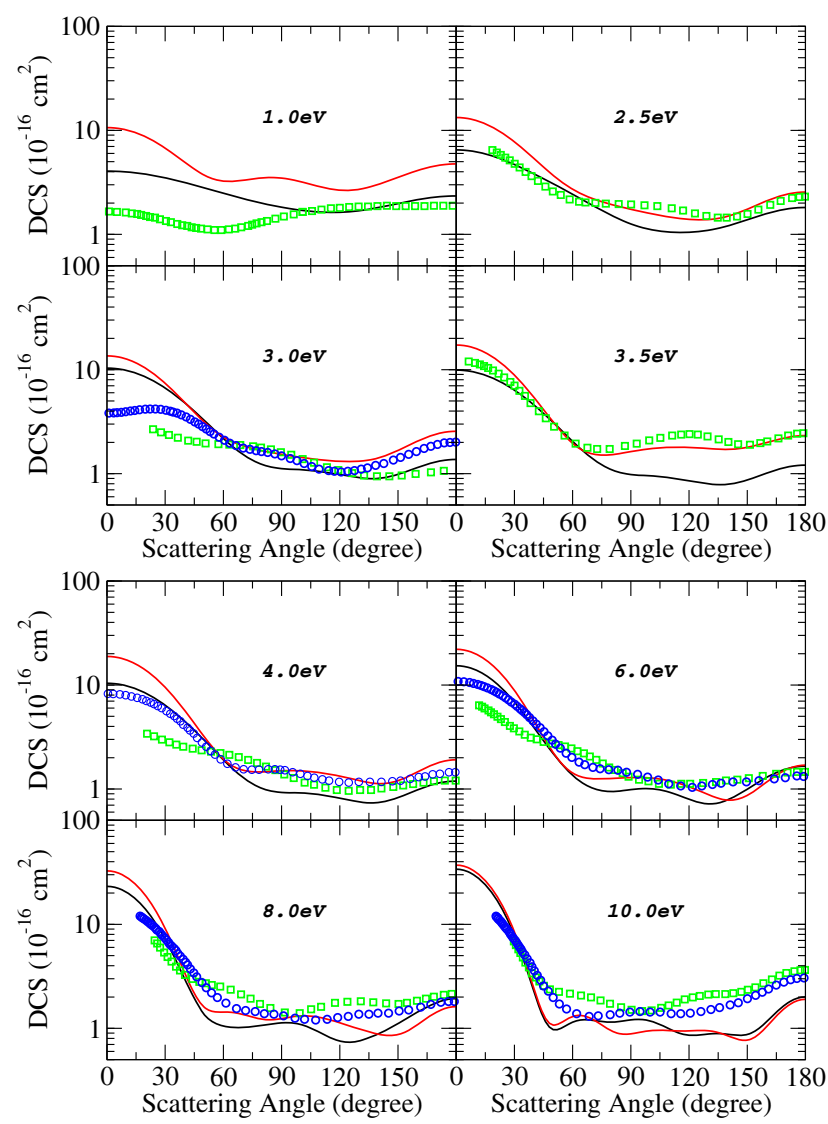

Fig. 6. SEP-DCS without Born correction for elastic electron scattering by alanine conformers $\mathrm{I}$ and $\mathrm{M}$ at impact energies ranging from $1 \mathrm{eV}$ to $10 \mathrm{eV}$; black line: this work, conformer I; red line: this work, conformer M. Also shown are theoretical results for glycine: squares: Schwinger multichannel (SMC) results [72]; circles: R-matrix results of Tashiro [71].

between 5 and $9 \mathrm{eV}$, which they indicated that could be associated to first and second excited state and were observed in almost all ions they studied. We find a broad resonance for conformers I and $\mathrm{M}$ at 9.8 and $8.6 \mathrm{eV}$, respectively, whose presence can also be seen in the cross sections presented in Figure 7 . Unlike the low energy resonance, it is not straightforward to be specific about the nature of this resonance feature. For glycine, Tashiro considered that the higher resonance could be related to core-excited states and dos Santos et al. speculated that a shape resonance observed at $9.5 \mathrm{eV}$ could be related to the sigma orbital located at hydroxyl group, but they not present any positive evidence for this.

Figure 8 compares our ICS for conformer I including Born correction with results of Marinkovic et al. [51] and Panosetti et al. [10]. Panosetti et al. calculated the ICS for the lowest energy molecular structure (which should be conformer I). The discrepancies between our results and of Marinković et al. can be attributed to the different method of calculation. They used the corrected independent-atom method (IAM) incorporating an improved quasi-free absorption model which is not expected to give a precise description of the electron-molecule colli-
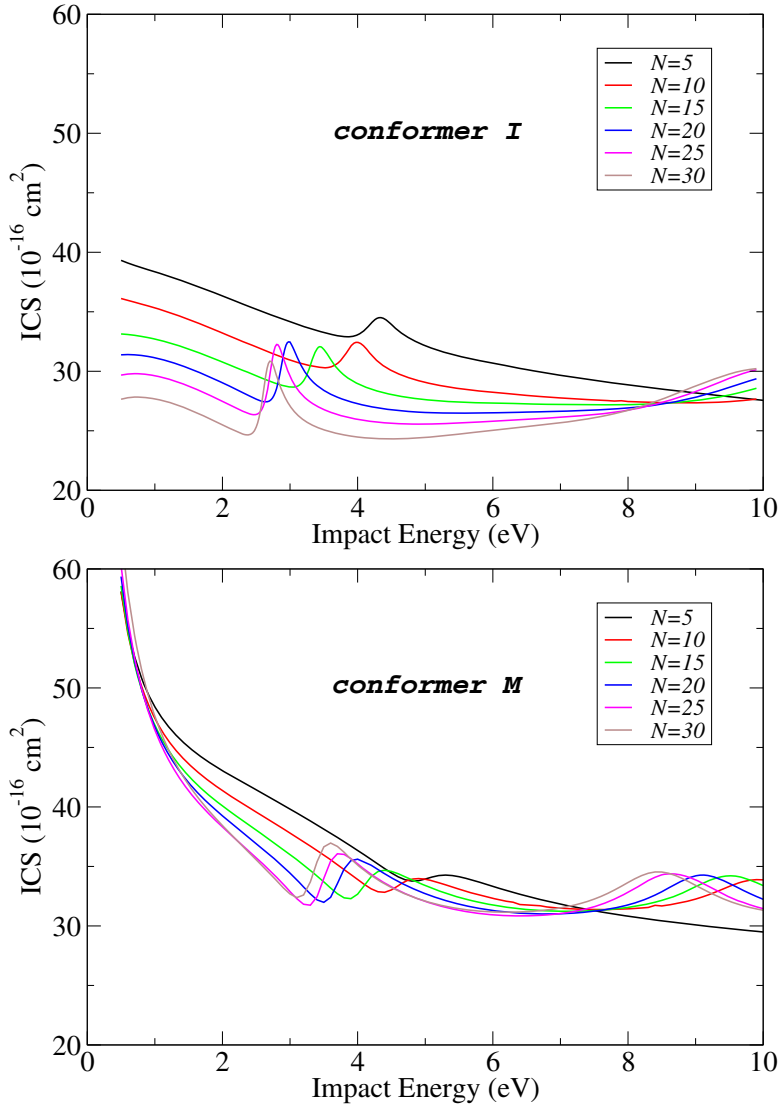

Fig. 7. Integral elastic cross section for electron scattering by alanine conformers I and $\mathrm{M}$ without Born correction: influence of polarization on the resonance positions: $N$ is the number of virtual orbitals used in the calculation.

sion at low impact energies. In this context, we note that a recent joint experimental-theory study on electron collisions with 1,4-dioxane [74] found good agreement with SEP calculations performed using the UKRMol codes up to energies of $20 \mathrm{eV}$ but less satisfactory agreement with IAM calculations. These findings are consistent with the fact that the IAM does not consider detailed structural information for the target which is important at for lowenergy electron collisions but become less so at higher energies. Despite this, our results seem to be in better agreement with Marinković et al. at impact energies lower than $4 \mathrm{eV}$ than with Panosetti et al. Below $2 \mathrm{eV}$ our ICS are bigger than the other results, which can be attributed to the way that we include Born correction.

The discrepancies observed between our results and of Panosetti et al. is slightly more puzzling but can probably be attributed to different levels of polarization in the calculation which can be gauged by different resonance peak positions. Panosetti et al. state that they use a potential model to represent the collisions which includes exchange, correlation and polarization effects. Panosetti et al. studied a series of amino acids: glycine, alanine, proline and valine; they state that for most of these amino acids the resonance energies are around $3 \mathrm{eV}$ but their figure for alanine shows the lower resonant structure near 


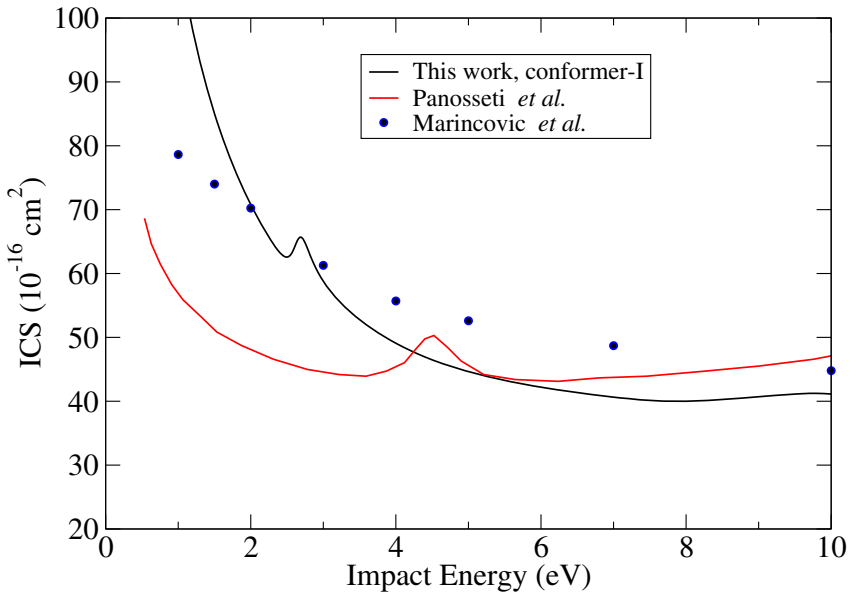

Fig. 8. Comparison of total cross section for elastic electron scattering by alanine conformer I: black line, this work; red line, Panosetti et al. [10]; circles: Marinković et al. [51].

$4.5 \mathrm{eV}$. This value is similar to ours $(4.5 \mathrm{eV})$ calculated at the static exchange level without polarization effects included. Similarly, they find a second resonance near $11 \mathrm{eV}$ while we find at $9.8 \mathrm{eV}$. The incomplete convergence of our eigenphase sums at this energy suggest that our value is almost certainly an upper limit for the true position of this resonance. Finally, our larger cross sections at energies below $4 \mathrm{eV}$ can be explained by their inclusion of partial waves up to 80 while our Born correction considers all partial waves.

\section{Conclusions}

We report a study of electrons scattered elastically by two alanine conformers in the gas phase for electron energies in the range 1 to $10 \mathrm{eV}$. The DCSs were calculated using R-matrix method and taking into account polarization effects at the SEP level retaining up to 30 virtual orbitals in the excitation space. The large dipole moment of alanine means that the dipole interaction, not the polarization potential, is the dominant long-range interaction, meaning that cross sections can be converged relatively easily in this model. However other properties, notably resonance positions, are more sensitive to short-range effects and it is hard to prove that our calculation completely converges the parameters of the two resonances we identify in this system. The R-matrix with pseudo-states (RMPS) method for molecules [75,76] offers some hope in performing such calculations in a systematic manner in which convergence of the polarization potential can be demonstrated [77]. However, despite technical advances [78], an RMPS calculation on the electron alanine problem remains too computationally demanding to be possible.

Despite the differences of a $\mathrm{CH}_{3}$ group and in the geometry of the main conformers, alanine and glycine have very similar structures. Although the permanent dipole moment of alanine conformer $\mathrm{M}$ is much larger than that of glycine, the comparison with available DCS results for glycine show magnitudes generally similar to our results for alanine when no Born correction is included. The differences observed at some energies (e.g., 1 eV) can be attributed to different size of molecules, conformations and dipole moments.

Our calculations find two resonances for each conformers: for conformer I we find one near $2.7 \mathrm{eV}$ and a broader one at about $9.8 \mathrm{eV}$, while conformer $\mathrm{M}$ shows a sharp resonance at $3.5 \mathrm{eV}$ and a broader one at about $8.6 \mathrm{eV}$. The lower 2.7 and $3.5 \mathrm{eV}$ resonance energy are associated with the $\pi^{*}$ unoccupied orbital of the carboxyl group. The broad resonances at 9.8 and $8.6 \mathrm{eV}$ cannot be easily assigned to a specific process, but it has been suggested [71] that it could arise from a core-excited states or $\sigma^{*}$ unoccupied orbital of $\mathrm{OH}$ group [50].

M.M.F. and S.E.M. acknowledge a grant from the Brazilian agency Conselho Nacional de Desenvolvimento Científico e Tecnológico (CNPq).

\section{References}

1. B. Boudaïffa, P. Cloutier, D. Hunting, M.A. Huels, L. Sanche, Science 287, 1658 (2000)

2. C. von Sonntag, The Chemical basis for radiation biology (Taylor and Francis, London, 1987)

3. I. Baccarelli, I. Bald, F.A. Gianturco, E. Illenberger, J. Kopyra, Phys. Rep. 508, 1 (2011)

4. L. Sanche, Eur. Phys. J. D 35, 367 (2005)

5. S. Tonzani, C.H. Greene, J. Chem. Phys. 125, 094504 (2006)

6. F. Blanco, G. García, Phys. Lett. A 360, 707 (2007)

7. P. Cloutier, C. Sicard-Roselli, E. Escher, L. Sanche, J. Phys. Chem. B 111, 1620 (2007)

8. T. Solomun, T. Skalicky, Chem. Phys. Lett. 453, 101 (2008)

9. L. Zhang, Z. Tan, Radiat. Environ. Biophys. 49, 15 (2010)

10. C. Panosetti, I. Baccarelli, F. Sebastianelli, F.A. Gianturco, Eur. Phys. J. D 60, 21 (2010)

11. S. Denifl, P. Sulzer, D. Huber, F. Zappa, M. Probst, T.D. Märk, P. Scheier, N. Injan, J. Limtrakul, R. Abouaf, H. Dunet, Angew. Chem. Int. Ed. 46, 5238 (2007)

12. C. Winstead, V. McKoy, Radiat. Phys. Chem. 77, 1258 (2008)

13. P. Papp, P. Shchukin, Š. Matejčík, J. Chem. Phys. 132, $014301(2010)$

14. Y.-F. Wang, S.X. Tian, J. Yang, Phys. Chem. Chem. Phys. 13, 15597 (2011)

15. M. Michaud, M. Bazin, L. Sanche, J. Chem. Phys. 137, 115103 (2012)

16. R. Barrios, P. Skurski, J. Simons, J. Phys. Chem. B 106, 7991 (2002)

17. R. Abouaf, J. Pommier, H. Dunet, Int. J. Mass Spectrom. 226, 397 (2003)

18. Z. Tan, Y. Xia, X. Liu, M. Zhao, Y. Ji, F. Li, B. Huang, Radiat. Environ. Biophys. 43, 173 (2004)

19. J. Berdys, P. Kurski, J. Simons, J. Phys. Chem. B 108, $5800(2004)$

20. J. Berdys, I. Anusiewicz, P. Kurski, J. Simons, J. Phys. Chem. A 108, 2999 (2004) 
21. L. Caron, L. Sanche, Phys. Rev. A 72, 032726 (2005)

22. J. Simons, Acc. Chem. Res. 39, 772 (2006)

23. C. Winstead, V. McKoy, J. Chem. Phys. 125, 174304 (2006)

24. C. Winstead, V. McKoy, S. d'A. Sanchez, J. Chem. Phys. 127, 085105 (2007)

25. C. Winstead, V. McKoy, Phys. Rev. A 76, 012712 (2007)

26. C. Winstead, V. McKoy, Phys. Rev. Lett. 98, 113201 (2007)

27. S.A. Pshenichnyuk, G.A. Gallup, P.D. Burrow, J. Phys. Chem. A 111, 11837 (2007)

28. C. Winstead, V. McKoy, J. Chem. Phys. 125, 244302 (2006)

29. P.D. Burrow, G.A. Gallup, A. Modelli, J. Phys. Chem. A 112, 4106 (2008)

30. A. Dora, L. Bryjko, T. van Mourik, J. Tennyson, J. Chem. Phys. 130, 164307 (2009)

31. C.J. Colyer, S.M. Bellm, F. Blanco, G. García, B. Lohmann, Phys. Rev. A 84, 042707 (2011)

32. A. Dora, L. Bryjko, T. van Mourik, J. Tennyson, J. Chem. Phys. 136, 024324 (2012)

33. A. Dora, L. Bryjko, T. van Mourik, J. Tennyson, J. Phys. B 45, 175203 (2012)

34. S. Ptasińska, S. Denifl, P. Scheier, T.D. Märk, J. Chem. Phys. 120, 8505 (2004)

35. C. König, J. Kopyra, I. Bald, E. Illenberger, Phys. Rev. Lett. 97, 018105 (2006)

36. W.M. Huo, C.E. Dateo, G.D. Fletcher, Radiat. Meas. 41, $1202(2006)$

37. I. Bald, J. Kopyra, I. Dabkowska, E. Antonsson, E. Illenberger, J. Chem. Phys. 126, 074308 (2007)

38. C. Winstead, V. McKoy, Int. J. Mass Spectrom. 277, 279 (2008)

39. C. Winstead, V. McKoy, J. Chem. Phys. 125, 074302 (2006)

40. B. Renjith, S. Bhowmick, M.K. Mishra, M. Sarma, J. Phys. Chem. A 115, 13753 (2011)

41. A. Gauf, L.R. Hargreaves, A. Jo, J. Tanner, M.A. Khakoo, T. Walls, C. Winstead, V. McKoy, Phys. Rev. A 85, 052717 (2012)

42. L. Bryjko, T. van Mourik, A. Dora, J. Tennyson, J. Phys. B 43, 235203 (2010)

43. O. Boulanouar, M. Fromm, C. Mavon, P. Cloutier, L. Sanche, J. Chem. Phys. 139, 055101 (2013)

44. D.F. Regulla, U. Deffner, Int. J. Appl. Radiat. Isot. 33, $1101(1982)$

45. V. Nagy, J.M. Puhl, M.F. Desrosiers, Radiat. Phys. Chem. 57, $1(2000)$

46. S. Ebraheem, W.B. Beshira, S. Eid, R. Sobhy, A. Kovacs, Radiat. Phys. Chem. 67, 569 (2003)

47. W.L. McLaughlin, D.M. Taylor, Appl. Radiat. Isot. 47, 1 (1996)

48. S. Ptasińska, S. Denifl, P. Candori, Š. Matejčík, P. Scheier, T.D. Märk, Chem. Phys. Lett. 403, 107 (2005)

49. I. Ipolyi, P. Cicman, S. Denifl, V. Matejčík, P. Mach, J. Urban, P. Scheier, T.D. Märk, Š. Matejčík, Int. J. Mass Spectrom. 252, 228 (2006)

50. A.M. Scheer, P. Mozejko, G.A. Gallup, P.D. Burrow, J. Chem. Phys. 126, 174301 (2007)
51. B.P. Marinković, F. Blanco, D. Šević, V. Pejčev, G. García, D.M. Filipović, D. Pavlović, N.J. Mason, Int. J. Mass Spectrom. 277, 300 (2008)

52. R. Abouaf, Chem. Phys. Lett. 451, 25 (2008)

53. A. Osted, J. Kongsted, O. Christiansen, J. Phys. Chem. A 109, $1430(2005)$

54. F. Ferreira da Silva, S. Denifl, T.D. Märk, A.M. Ellis, P. Scheier, J. Chem. Phys. 132, 214306 (2010)

55. C.J. Gillan, J. Tennyson, P.G. Burke, in Computational methods for Electron-molecule collisions, edited by W. Huo, F.A. Gianturco (Plenum, 1995), pp. 239-254

56. J. Tennyson, Phys. Rep. 491, 29 (2010)

57. N.T. Padial, D.W. Norcross, L.A. Collins, J. Phys. B 14, 2901 (1981)

58. M.A. Morrison, Adv. At. Mol. Phys. 24, 51156 (1988)

59. F.A. Gianturco, A. Jain, Phys. Rep. 143, 347 (1986)

60. N. Sanna, F.A. Gianturco, Comput. Phys. Commun. 114, $142(1998)$

61. J. Tennyson, D.B. Brown, J.J. Munro, I. Rozum, H.N. Varambhia, N. Vinci, J. Phys.: Conf. Ser. 86, 012001 (2007)

62. J.M. Carr, P.G. Galiatsatos, J.D. Gorfinkiel, A.G. Harvey, M.A. Lysaght, D. Madden, Z. Mašin, M. Plummer, J. Tennyson, H.N. Varambhia, Eur. Phys. J. D 66, 58 (2012)

63. S. Blanco, A. Lesarri, J.C. López, J.L. Alonso, J. Am. Chem. Soc. 126, 11675 (2004)

64. National Institute of Standards and Technology, Standard Reference Data at http://cccbdb.nist.gov

65. M. Bode, M.S. Gordon, J. Mol. Graph. Model. 16, 133 (1998)

66. P.D. Godfrey, S. Firth, L.D. Hatherley, R.D. Brown, A.P. Pierlot, J. Am. Chem. Soc. 115, 9687 (1993)

67. A. Faure, J.D. Gorfinkiel, L.A. Morgan, J. Tennyson, Comput. Phys. Commun. 144, 224 (2002)

68. G. Danby, J. Tennyson, J. Phys. B 23, 1005 (1990)

69. J. Tennyson, C.J. Noble, Comput. Phys. Commun. 33, 421 (1984)

70. M.M. Fujimoto, W.J. Brigg, J. Tennyson, Eur. Phys. J. D 66, 204 (2012)

71. M. Tashiro, J. Chem. Phys. 129, 164308 (2008)

72. J.S. dos Santos, R.F. da Costa, M.T. do N. Varella, J. Chem. Phys. 136, 084307 (2012)

73. K. Aflatooni, B. Hitt, G.A. Gallup, P.D. Burrow, J. Chem. Phys. 115, 14 (2001)

74. P. Palihawadana, J.P. Sullivan, S.J. Buckman, Z. Masin, J.D. Gorfinkiel, F. Blanco, G. Garcia, M.J. Brunger, J. Chem. Phys. 139, 014308 (2013)

75. J.D. Gorfinkiel, J. Tennyson, J. Phys. B 37, L343 (2004)

76. J.D. Gorfinkiel, J. Tennyson, J. Phys. B 38, 1607 (2005)

77. M. Tarana, J. Tennyson, J. Phys. B 43, 045101 (2010)

78. R. Zhang, P.G. Galiatsatos, J. Tennyson, J. Phys. B 44, 195203 (2011)

Open Access This is an open access article distributed under the terms of the Creative Commons Attribution License (http://creativecommons.org/licenses/by/4.0), which permits unrestricted use, distribution, and reproduction in any medium, provided the original work is properly cited. 